\title{
Supercontinuum Generation in a Silica Spiral Waveguide
}

\author{
Dong Yoon Oh ${ }^{1}$, David Sell ${ }^{1}$, Hansuek Lee ${ }^{1}$, Ki Youl Yang ${ }^{1}$, Scott A. Diddams ${ }^{2}$, Kerry J. Vahala ${ }^{1}$ \\ ${ }^{1}$ T. J. Watson Laboratory of Applied Physics, California Institute of Technology, Pasadena, CA 91125, USA \\ ${ }^{2}$ Time and Frequency Division, National Institute of Standards and Technology, Boulder, Colorado 80305, USA \\ Authore-mail address: vahala@caltech.edu
}

\begin{abstract}
A low-loss silica spiral waveguide is used for demonstrating on-chip supercontinuum generation. The broadest measured spectrum spans an octave $(936-1888 \mathrm{~nm})$ at $-50 \mathrm{~dB}$ from peak when $2.17 \mathrm{~nJ}$ pulses are launched.
\end{abstract}

OCIS codes: (320.6629) Supercontinuum generation, (190.4390) Nonlinear optics, integrated optics

Supercontinuum generation( $\mathrm{SCG}$ ) has been previously reported in chip-based systems including silicon photonic nanowires [1], chalcogenide waveguides [2] and silicon nitride $\left(\mathrm{Si}_{3} \mathrm{~N}_{4}\right)$ waveguides [3]. Historically, fiber-based SCG devices have found a broad range of applications including frequency combs [4], optical coherence tomography [5], and wavelength division multiplexing [6]. In such context, on-chip waveguides for SCG would bring important functionalities of photonic integrated circuits. Although silica has a small Kerr coefficient of $2.6 \times$ $10^{-20} \mathrm{~m}^{2} \mathrm{~W}^{-1}$, it has a much lower linear loss compared to other materials, a crucial factor for the success of fiber based devices. Recently, a chip-based, silica waveguide functioning as a low-loss optical delay line was reported [7]. Here, the ability for SCG using the waveguide is tested.
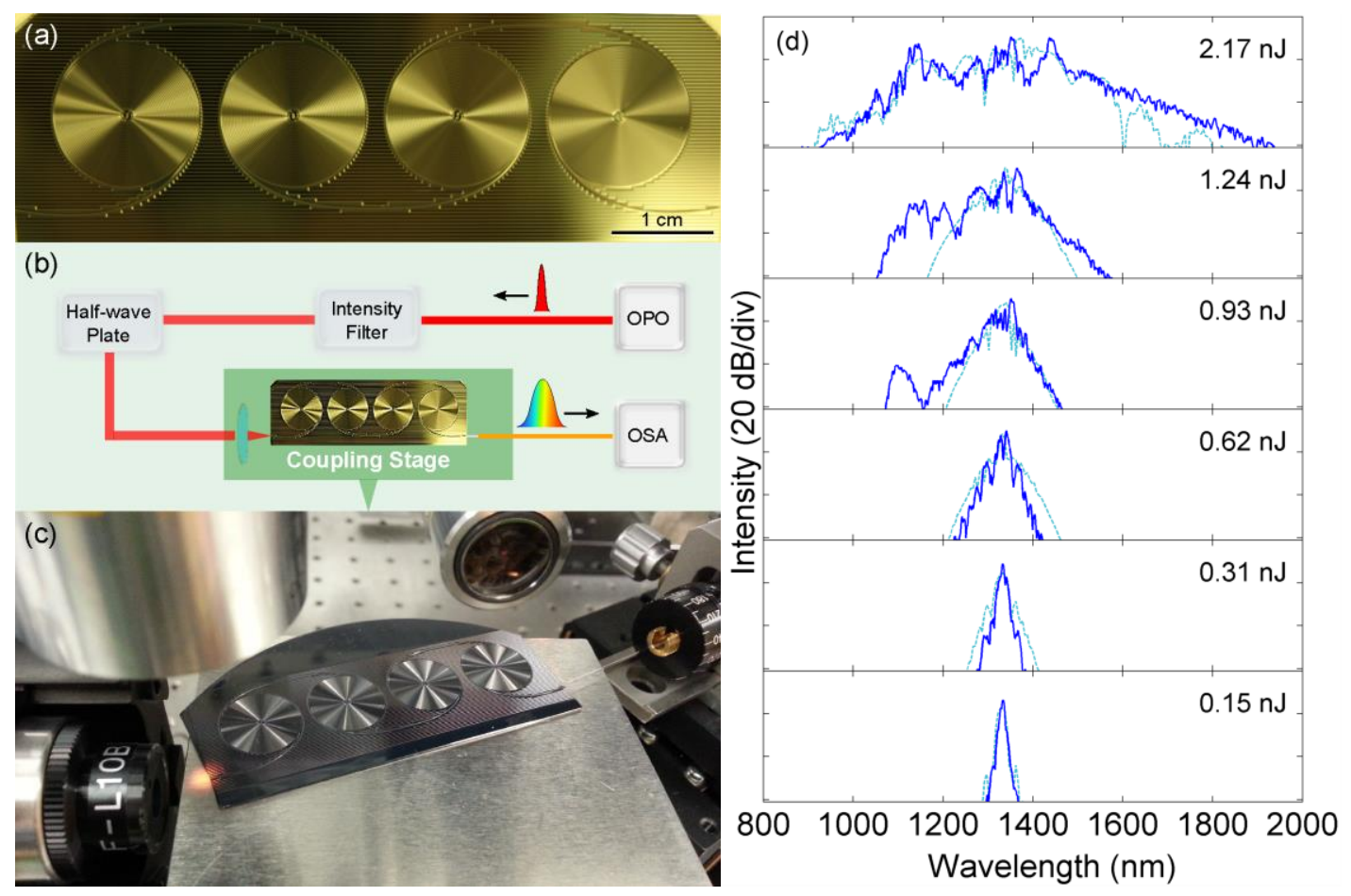

Fig. 1. (a) Photograph of the sample showing four, cascaded spiral waveguides. (b) Experimental setup for supercontinuum generation and characterization using the silica waveguide. OPO: optical parametric oscillator; OSA: optical spectrum analyzer. (c) Close-up view of the optical coupling stage, imaging objectives and spiral waveguide chip. (d) Spectral broadening in the fundamental TM mode measured at a series of pulse energies. The blue curves are measured spectra, and the dashed curves in light blue are spectra from the simulation.

A silica waveguide with a total physical path length of $3.5 \mathrm{~m}$ consisting of four, cascaded, double-interleaved Archemidian spirals is used in the experiment (Fig. 1a). The outer radius of each spiral is $7.0 \mathrm{~mm}$ and the chip size is $2.5 \mathrm{~cm} \times 6.9 \mathrm{~cm}$. A pulse train with $80-\mathrm{MHz}$ repetition rate and FWHM of $180 \mathrm{fs}$ is launched from an optical parametric oscillator (Spectra-Physics OPAL). The energy and the polarization of the pulse are controlled using a variable neutral density filter and a half-wave plate, as shown in Fig. 1b. An objective lens with 12-mm focal length is used to free-space couple the light into the waveguide. At the waveguide end, a cleaved multimode fiber is closely 
positioned to receive the light from the waveguide. The coupling loss is estimated to be around $3 \mathrm{~dB}$ at the input and $10 \mathrm{~dB}$ at the output. Two OSAs were used to separately record the spectra in the wavelength range $600-1700 \mathrm{~nm}$ (Agilent 86141B) and 1200-2400 nm (Yokogawa AQ6375). As the center wavelength of the input pulse is varied from $1250 \mathrm{~nm}$ to $1350 \mathrm{~nm}$, the broadest spectrum is observed near $1330 \mathrm{~nm}$. Measured TM mode spectra for various coupled pulse energies are presented in Fig. 1d. At the maximum energy of $2.17 \mathrm{~nJ}$, an octave spanning continuum extending from $936 \mathrm{~nm}$ to $1888 \mathrm{~nm}(162 \mathrm{THz})$ at $-50 \mathrm{~dB}$ from peak is generated. Both TE and TM mode SCG were studied and exhibited qualitatively similar behavior both in the measurements and modeling. However, only data for the TM modes are presented as their spectra are slightly broader on account of a reduced modal, crosssectional area relative to the TE modes.

The pulse propagation in the waveguide is modeled by numerically solving a generalized nonlinear Schrödinger equation in a split-step fashion [8]. For this calculation, the dispersion and nonlinearity of the waveguide are calculated using a finite element solver (COMSOL) at twenty-two radii that can occur inside the waveguide. The quantities are interpolated at all the other radii of the waveguide. The resulting spectra agree well with the measured ones (See Fig. 1d).

In conclusion, we demonstrated a SCG in a low-loss silica waveguide using femtosecond pulses from an OPO. The broadest spectrum in TM modes spans an octave. Good agreement between the measured spectra and those from a simulation has been found.

\section{References}

[1] I. W. Hsieh, X. Chen, X. Liu, J. I. Dadap, N. C. Panoiu, C. Y Chou, F. Xia, W. M. Green, Y. A. Vlasov,and R. M. Osgood, "Supercontinuum generation in silicon photonic wires", Opt. Express 15, 15242 (2007).

[2] M. R. Lamont, B. L. Davies, D. Y. Choi, S. Madden, and B. J. Eggleton, "Supercontinuum generation in dispersion engineered highly nonlinear $(\gamma=10 / \mathrm{W} / \mathrm{m}) \mathrm{As}_{2} \mathrm{~S}_{3}$ chalcogenide planar waveguide", Opt. Express 16, 14938 (2008).

[3] R. Halir, Y. Okawachi, J. S. Levy, M. A. Foster, M. Lipson, and A. L. Gaeta, "Ultrabroadband supercontinuum generation in a CMOS-compatible platform", Opt. Lett. 37, 1685 (2012).

[4] S. A. Diddams, D. J. Jones, J. Ye, S. T. Cundiff, J. L.Hall, J. K. Ranka, R. S. Windeler, R. Holzwarth, T. Udem, and T. W. Hänsch, "Direct Link between Microwave and Optical Frequencies with a 300 THz Femtosecond Laser Comb”, Phys. Rev. Lett. 84, 5102 (2000).

[5] I. Hartl, X. D. Li, C. Chudoba, R. K. Ghanta, T. H. Ko, J. G. Fujimoto, J. K. Ranka and R. S. Windeler, "Ultrahigh-resolution optical coherence tomography using continuum generation in an air-silica microstructure optical fiber” Opt. Lett. 26, 208 (2001).

[6] S. Kawanishi, H. Takara, K. Uchiyama, I. Shake, and K. Mori, “3Tbit/s (160Gbit/s x 19 channel) optical TDM and WDM transmission experiment”, Electron. Lett. 35, 826 (1999).

[7] H. Lee, T. Chen, J. Li, O. Painter, and K. J. Vahala, "Ultra-low-loss optical delay line on a silicon chip”, Nature Commun. 3, 867 (2012).

[8] J. M. Dudley, and J. R. Taylor, Supercontinuum Generation in Optical Fibers (Cambridge University, 2010), Chap. 3. 